Ebisu Ebisu

Études japonaises Études japonaises

$53 \mid 2016$

1914-1918, une guerre mondiale? La perspective

japonaise

\title{
Shiramine: drame musical en trois actes et douze scènes
}

\section{Akira Tamba}

Traducteur : Julia Saint-Pol, Jean-Michel Butel, Brigitte Allioux et Jean-Jacques Tschudin

\section{(2) OpenEdition}

\section{Journals}

Édition électronique

URL : https://journals.openedition.org/ebisu/1931

DOl : 10.4000/ebisu.1931

ISSN : 2189-1893

\section{Éditeur}

Institut français de recherche sur le Japon à la Maison franco-japonaise (UMIFRE 19 MEAE-CNRS)

\section{Édition imprimée}

Date de publication : 10 décembre 2016

Pagination : 239-270

ISSN : 1340-3656

\section{Référence électronique}

Akira Tamba, «Shiramine : drame musical en trois actes et douze scènes », Ebisu [En ligne], 53 | 2016,

mis en ligne le 10 décembre 2016, consulté le 08 novembre 2021. URL : http://

journals.openedition.org/ebisu/1931; DOI : https://doi.org/10.4000/ebisu.1931 


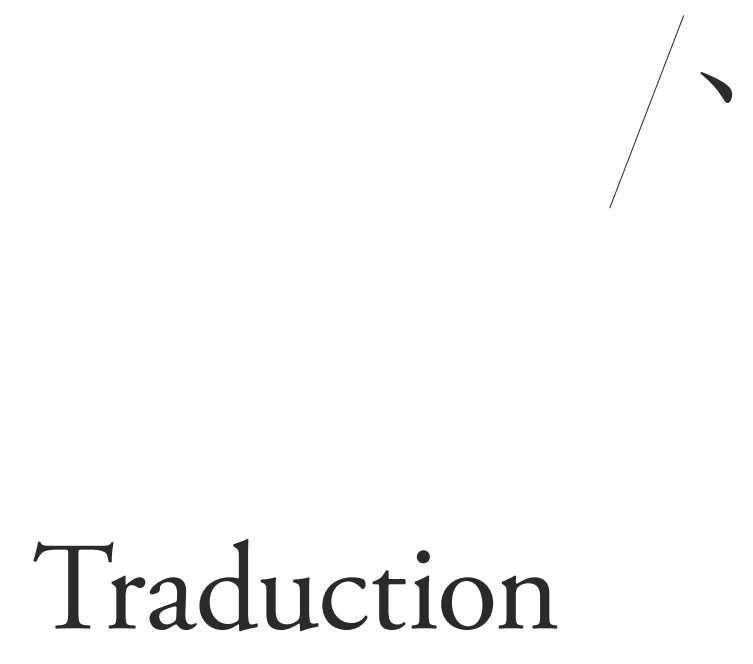





\title{
Shiramine
}

\section{Drame musical en trois actes et douze scènes}

\author{
Akira TAMBA 丹波明*
}

L'opéra Shiramine, écrit et composé par Akira Tamba, a été créé au Sumida Triphony Hall le 28 septembre 2014. Les derniers réglages ont eu lieu à la Maison franco-japonaise, où le compositeur résidait. Nous sommes heureux de pouvoir présenter dans les pages qui suivent le livret de cet opéra, précédé d'une explication du compositeur sur la genèse de son ouvre et les principes esthétiques qui l'ont inspiré.

\section{Retour sur la genèse de l'opéra Shiramine}

L'histoire de Shiramine commence il y a 12 ou 13 ans avec la rencontre de deux hommes de lettres qui m'incitèrent à composer un opéra ${ }^{1}$. Nourrissant depuis quelque temps déjà le projet d'écrire une trilogie, je fus tout de suite intéressé par leur proposition. À cette époque, je souhaitais créer une œuvre

1. 《Gakugeki Shiramine tanjōtan » 楽劇「白峰」誕生票, Higeki kigeki 悲劇喜劇, $\mathrm{n}^{\circ} 724$, 2011-2, p. 19-21. Nous remercions la revue Higeki kigeki d'en avoir permis la traduction. Toutes les notes de cette version française sont des traducteurs.

* Compositeur, musicologue. 
ayant pour thème les trois religions que sont le christianisme, le bouddhisme et l'islam, et qui considérerait leurs interactions avec chaque société, avec chaque culture, ainsi que leurs implications sur la vie et les émotions quotidiennes, sur la pensée et les drames humains qu'elles provoquent. L'un des deux hommes évoqua la version de Shiramine des Contes de pluie et de lune $e^{2}$. Je lus alors cette nouvelle et j'acceptai immédiatement l'idée. Le texte, qui suivait la forme des nô d'apparition ${ }^{3}$, me permettait en effet d'appliquer le concept de jo-ha-kyū sur lequel j'ai beaucoup travaillés. Il suscita par ailleurs chez moi toutes sortes d'idées sur plusieurs plans. Nous convînmes que l'un des partenaires prendrait en charge le premier et le deuxième acte, et que l'autre s'occuperait du troisième. Nous répartîmes les rôles en décidant que le premier et le troisième actes suivraient les principes des nô d'apparition, tandis que le second prendrait la forme d'un nô du monde réel ${ }^{5}$. Avant que le livret ne me soit envoyé, je terminai le prélude, la scène suivante durant laquelle le moine Saigyō lit les sûtras, ainsi que la scène où Saigyō et le bûcheron (en réalité l'esprit de l'empereur décédé Sutoku) échangent leurs noms, et c'est, à l'inverse de ce qui était prévu, finalement moi qui transmis aux scénaristes un texte rédigé en me conformant aux règles de présentation d'un personnage dans le nô. Quelques semaines plus tard, je reçus la première partie de l'acte I. Il s'agissait d'un

2. Ueda Akinari 上田秋成, Ugetsu monogatari 雨月物語 (1776), traduit, présenté et annoté par René Sieffert, Contes de pluie et de lune, Paris, Gallimard, 1956. Shiramine est la première nouvelle du recueil.

3. Le nô d'apparition (mugen nō 夢幻能) met en scène l'apparition d'un personnage surnaturel tel qu'un dieu, un démon ou encore un fantôme ; Zeami, La Tradition secrète du nô suivi d'Une Journée de nô, traduit du japonais par René Sieffert, Paris, Gallimard, coll. Connaissance de l'Orient, 1960.

4. Le jo-ha-kyū 序破急 (littéralement "introduction, briser, rapide ») est un principe de mutation progressive utilisé dans les arts traditionnels et notamment le nô où il a été introduit par Zeami 世阿弥. Il consiste pour celui-ci en trois mouvements : jo renvoie à l'introduction (il correspond, dans la plupart des cas, à l'entrée en scène du waki 脇), ha est le développement, durant lequel le shite 仕手 raconte son histoire, tandis que kyū marque la conclusion. Akira Tamba a souvent présenté cette notion, par exemple en français dans La théorie et l'esthétique musicale japonaise du $8^{e}$ siècle à la fin du $19^{e}$ siècle, Paris, Presses orientalistes de France, 1992, ou La musique classique du Japon du XV siècle à nos jours, Paris, Presses orientalistes de France, 2001, p. 11-14.

5. Un nô du monde réel (genzai nō 現在能) met en scène les sentiments humains dans une situation tragique. Zeami, op. cit. 
long récitatif $\mathrm{du}$ bûcheron entrecoupé parfois de deux ou trois mesures d'accompagnement jouées par l'orchestre afin d'offrir quelques pauses au chanteur, le tout occupant environ cinquante minutes. Il me fallut demander un certain nombre de modifications : faire intervenir Saigyō trois ou quatre fois pour transformer la scène en dialogue ${ }^{6}$, tenter de remplacer par des synonymes à la prosodie ascendante les mots à la prosodie descendante qui tombaient aux endroits où la mélodie elle-même montait, raccourcir ou rallonger les phrases lorsque celles-ci ne coïncidaient pas avec la mélodie, modifier l'ordre des phrases lorsqu'elles ne s'accordaient pas avec le principe du jo-ha-kyù.

Les librettistes acceptèrent tout d'abord mes modifications et proposèrent des solutions convenables, mais sans doute furent-ils ensuite débordés par mes demandes successives, car ils se retirèrent du projet. Pourtant mes requêtes n'étaient pas uniquement dues à mon entêtement, mais à une exigence de la musique elle-même. Il faut comprendre la différence qui existe entre le livret d'un opéra et celui d'une pièce de théâtre. Un opéra suit une structure musicale. Les éléments littéraires sont des matériaux qui guident le rythme de la musique, sa vitesse, sa mélodie, sa tonalité (triste, mystérieuse, gaie...). La structure musicale est issue ensuite d'un second processus de création qui fait appel à la sensibilité propre du compositeur. Au théâtre, à l'inverse, les scénaristes s'assurent de la structure de la pièce. S’il y a ajout éventuel de musique, celle-ci conserve la structure théâtrale et intervient comme un matériau psychologique venant renforcer l'effet dramatique. Elle joue un rôle d' " accompagnement musical ». Les conflits entre librettistes et compositeurs ont souvent pour cause la question du matériau souverain qui doit structurer l'œuvre. C'est un problème que beaucoup de compositeurs et d'écrivains ont déjà vécu. Ce fut le cas par exemple lors de l'écriture de Pelléas et Mélisande, qui mena Debussy et Maeterlinck jusqu'au procès. Afin d'éviter ce genre de conflits, de nombreux compositeurs en sont venus à écrire eux-mêmes le livret en même temps que la musique. Wagner ou Messiaen en sont de bons exemples.

C'est déjà ce qu'avait suggéré au XIv siècle Zeami Motokiyo 世阿弥元清 (1363-1443). Cet acteur de nô, qui a laissé de nombreux ouvrages théoriques sur son art, préconisait qu'une seule et même personne se charge

6. Ce qui est plus conforme à la fois au texte original et à la structure d'un nô. 
du scénario et de la musique. Dans son traité sur la Composition $d u$ nô (Nōsakusho 能作書, 1423), il explique :

La composition d'une pièce de nô repose sur trois éléments : la matière, la disposition et l'écriture. Il faut connaître sa matière, savoir la disposer, et la rédiger de manière adéquate. On doit d'abord bien maîtriser l'histoire que l'on souhaite traiter, puis la disposer en cinq séquences respectant les trois mouvements jo, ha et kyü, et enfin écrire en choisissant les mots et la musique qui conviennent à l'ensemble.

能は種、作、書の三道より出たり。一に能の種を知ること。二に能を作ること。 三に能を書くこと也。本説の種をよくよく案得して、序・破・急の三体に五段に 作りなして、さて詞を集めて曲を付けて書き連めるなり。

Zeami précise qu'il est indispensable de franchir ces trois étapes pour concevoir et composer une pièce de nô. Il s'agit dans un premier temps de connaître parfaitement le sujet développé dans le morceau. Dans un second temps de structurer le sujet afin de le faire correspondre aux trois mouvements jo-ha-kyū. Enfin, de réunir les textes, poèmes et maximes en rapport avec le sujet et de poursuivre la rédaction avec ces éléments tout en y joignant la musique.

Il faut ajouter quelques explications à propos des « trois mouvements jo, ha et kyū" qu'évoque Zeami pour décrire la seconde étape. Il s'agit d'un principe de base de l'esthétique traditionnelle japonaise qui tente d'organiser la structure des arts à fondement temporel ${ }^{7}$ par une augmentation graduelle des stimulations. Les stimulations dont il est question ici sont la hauteur des sons, la vitesse, l'intensité ou encore la densité de la musique (le nombre de sons). Zeami explique que c'est par l'augmentation progressive de ces paramètres qu'il faut façonner un morceau. Nous comprenons de façon très claire ici que la structure du nô est une structure musicale. Pour mon opéra Shiramine j'ai dû exiger des auteurs qu'ils transforment les textes, le rythme et les mots afin de les ajuster au principe du jo-ha-kyü, ce qui a été le point de départ du conflit.

C'est à cette période que je reçus du second librettiste le début de l'acte III. Ce troisième acte correspondant au kyū du jo-ha-kyū, les phrases se doivent d'être plus courtes et rythmiques. Or, le livret qui m'avait été

7. Lauteur reprend ici la distinction que fit Lessing dans son Laocoon (1766) entre arts du temps et arts de l'espace. 
envoyé était écrit dans un style $k a y \bar{o}-k y o k u^{8}$ qui faisait craindre que l'acte devînt trop long, et dont on pouvait douter de la cohérence avec le style d'écriture épique du premier librettiste. J'abandonnai donc la composition de la musique de Shiramine pour entreprendre la rédaction de l'Esthétique du jo-ha-kyū à laquelle je voulais me consacrer depuis quelque temps déjà. Le livre a été publié en 2004 par la maison d'édition Ongaku no tomosha ${ }^{9}$. Durant les trois années de "refroidissement " qui furent nécessaires à sa rédaction, je pris la résolution d'écrire moi-même le texte en parallèle avec la musique, comme le conseillait Zeami, et pour cela de réunir les documents nécessaires. Nommément les Contes de pluie et de lune, le Dit de $H_{o ̈ g e n}{ }^{10}$, le Gukanshō ${ }^{-11}$ et l'Ögishō ${ }^{-12}$.

\section{Style littéraire}

La langue japonaise actuelle étant très éloignée de la langue de la cour de la fin de la période Heian, j'ai imité la phraséologie ancienne et corrigé mes textes en me référant au style d'écriture des textes de nô (yōkyoku) et des Contes de pluie et de lune.

8. Le kayo-kyoku 歌謡曲 est un style musical populaire moderne. Le terme a tout d'abord été utilisé pour désigner la musique occidentale apparue au Japon durant l'ère Meiji mais a changé de signification durant l'ère Shōwa pour désigner la musique populaire " japonaise ".

9. Tamba Akira 丹波明, Jo-ha-kyū to iu bigaku. Gendai ni yomigaeru Nihon ongaku no shikōkei「序破急」という美学一現代によみがえる日本音楽の思考型 (Esthétique du jo-hakyü. Regards rétrospectifs sur la structure de la musique japonaise), Ongaku no tomosha 音楽之友社, 2004.

10. Högen monogatari 保元物語 (1320). Le Dit de Hôgen. Le Dit de Heiji. Cycle épique des Taïra et des Minamoto, I, trad. René Sieffert, Paris, Publication orientalistes de France, 1976, rééd. Verdier/poche, 2007.

11. Chronique historique attribuée au moine Jien 慈円 et achevée aux environs de 1220. Voir la traduction anglaise de Delmer Myers Brown \& Ichirō Ishida, The Future and the Past: A Translation and Study of the Gukanshō, An Interpretative History of Japan Written in 1219, Berkeley, University of California Press, 1979.

12. Ogishō 奥義抄 (1124-1144). Ouvrage de poétique rédigé par Fujiwara no Kiyosuke 藤原清輔 (1104-1177). 


\section{Construction}

En me conformant à la structure du jo-ha-kyū, j'ai réparti les douze scènes en trois actes, et organisé chaque acte en me conformant aux trois mouvements jo, ha et kyü (trois scènes pour l'acte I, sept scènes pour l'acte II, et deux scènes pour l'acte III).

\section{Mélodie}

La musique ne recourt pas à un développement mélodique de type aria. J'ai unifié le chant des protagonistes en adoptant pour tous la forme d'un récitatif fondé sur un intervalle de quarte dont j'ai modulé le degré (plus haut ou plus bas) et la vitesse (plus rapide ou plus lente) en fonction de chaque personnalité, de la situation, de la psychologie ou des sentiments des personnages. J'ai essayé de rendre le caractère affectif de ces changements en jouant avec ces différents paramètres et en insérant, avant ou après le motif musical, un motif qui permet d'identifier un personnage comme la nourrice, des sentiments intimes ou des thèmes dramatiques, comme le destin ou la guerre par exemple.

\section{Orchestration}

Il s'agit d'un orchestre symphonique auquel j'ai ajouté deux ondes Martenot afin de renouveler le volume des sons et leur timbre. Le chœur comprend environ soixante membres, qui se répartissent en fonction des moments en quatre, six ou huit groupes. L'utilisation d'un synthétiseur électronique à la fin du premier acte et au début du troisième, ainsi que la transformation progressive de la voix de l'empereur Sutoku qui peu à peu passe par un micro et des enceintes, créent une atmosphère fantastique et irréelle qui permet de différencier les hommes du monde réel de ceux de l'autre monde.

\section{Contenu}

Stratagèmes et complots à l'ombre de la splendeur de la cour de l'époque Heian. Entraîné dans ces engrenages, l'empereur Sutoku a été exilé sur l'île lointaine de Shikoku. Il y vivra, jusqu'à sa mort, quarante-cinq années d'une existence solitaire et malheureuse. Sa rancœur est restée profonde. 
Son âme tourmentée se réincarne dans un bûcheron, qui relate sa haine profonde au moine Saigyō, venu de la capitale afin de prier pour lui. Le second acte est une recomposition libre basée sur un fait historique, où sont représentés des personnages et des épisodes qui ont marqué l'histoire et la littérature japonaises : le premier empereur retiré Shirakawa ; le mariage de l'empereur Toba, père de Sutoku, avec sa première épouse Taikenmon-in ; la relation entre cette dernière et l'empereur Shirakawa ; la naissance (représentée par un berceau) de l'empereur Konoe, né de l'empereur Toba et de sa seconde épouse Bifukumon-in ; l'amour de cette dernière pour son fils, cause de la plus impitoyable guerre civile jamais vue au Japon ; la rébellion de Hōgen ${ }^{13}$; la chute et l'exil de Sutoku qui s'ensuivit.

$\mathrm{Au}$ troisième acte, l'esprit de Sutoku apparaît pour la seconde fois et déplore auprès de Saigyō le traitement inhumain, contraire aux lois du ciel, qui lui a été infligé par Go-Shirakawa et Bifukumon-in. Il explique alors qu'il a choisi la voie des icchantika ${ }^{14}$, qu'il est devenu un grand démon sans espoir de salut, un esprit malfaisant qui n'a de cesse de se venger, puis dévoile sa véritable apparence, celle d'un Sutoku entouré de grandes flammes éclatantes. Saigyō se réveille et, comprenant que tout ce qu'il vient de voir n'était qu'un affreux cauchemar, se livre de façon renouvelée à la lecture des sûtras. Le chœur se joint à lui pour clore l'acte.

Traduit par Julia Saint-Pol et Jean-Michel Butel

13. Guerre civile survenue durant la période Heian entre les partisans de l'empereur Sutoku et ceux de l'empereur Go-Shirakawa afin de décider de la succession impériale. Voir Le dit de Hôgen..., op. cit.

14. Dans le bouddhisme Mahayana, être dont le karma est si mauvais qu'il ne peut espérer accéder au salut. 


\section{Résumé et personnages}

\section{Résumé}

Acte I, scène I. Prologue: devant le mausolée abandonné de l'empereur Sutoku, exilé à Shiramine dans la province de Sanuki.

Le révérend moine Saigyō récite le sûtra d'Amida pour la rédemption des morts, devant la tombe de l'empereur Sutoku, dont il connaissait bien la famille. Saigyō, en effet, avait été attaché à la garde impériale avant de devenir moine pour apaiser son âme tourmentée.

\section{Acte I, scène II. Toujours devant le même mausolée.}

Tandis que Saigyō continue à réciter des sûtras, un bûcheron arrive par le fond de la scène en chantant. Ce bûcheron n'est autre que la réincarnation de l'empereur Sutoku. Questionné par Saigyō, il relate avec sérénité la vie de Sutoku. Né en 1119, Sutoku est le premier fils de l'empereur Toba et de l'impératrice Taikenmon-in. Son aïeul l'Empereur retiré, le pieux Shirakawa, qui lui portait une grande affection, le fit monter sur le trône à l'âge de cinq ans. Ainsi devint-il le soixante-quinzième empereur, en lieu et place de son père, l'empereur Toba. Mais, à la mort de Shirakawa, Toba, qui gouvernait en fait le pays, destitua Sutoku et mit sur le trône Konoe, alors âgé de deux ans, qui était le fils de sa seconde épouse Bifukumon-in. Malheureusement, Konoe mourut à l'âge de seize ans, à la suite, dit-on, d'un sort jeté par Sutoku. L'empereur retiré Toba éloigna alors du trône le fils de Sutoku, le prince Shigehito, au profit de Masahito, le frère cadet de Sutoku. L'empereur Sutoku perdait ainsi tout droit de lignage. D'où le terrible conflit de succession qui éclata à la mort de Toba, dans la première année de l'ère Hōgen, entre le clan de l'impératrice Bifukumon-in et celui de Sutoku. Ce fut la guerre civile de Hōgen, qui se termina par la défaite de l'empereur Sutoku et son exil sur l'île de Naoshima, dans la province de Sanuki.

C'est là que Sutoku entreprit de copier avec son propre sang cinq sûtras du Grand Véhicule afin d'obtenir la rédemption des âmes des soldats morts pour lui à la guerre. Il mit trois ans à les terminer et il les envoya à la capitale, accompagnés de poèmes, dans son souhait ardent que ces morts accèdent à la bouddhéité. Cependant, les femmes du Palais, craignant une malédiction, les rejetèrent et les renvoyèrent à Shiramine. Désespéré, 
Sutoku les enfouit profondément dans la mer, où ils devinrent des démons belliqueux Ashura, transformant ce bas monde en un lieu infernal. Il jura alors devant toutes les divinités de se venger. Suivirent neuf années de terribles souffrances, au terme desquelles l'empereur mourut. Sa haine était si grande, dit-on, que la fumée de sa crémation s'étendit en longues et horribles volutes en direction de la capitale.

Tel fut le récit du bûcheron.

\section{Acte I, scène III. Toujours devant la même tombe.}

Saigyō demande alors au bûcheron: "Comment savez-vous tant de choses sur la vie de l'empereur Sutoku ? - C'est, répond le bûcheron, que je suis la réincarnation de Sutoku. "Et, à ces mots, il se transforme sur le champ en un horrible démon et s'écrie : "Il est totalement vain et insensé de vouloir me faire devenir bouddha par la vertu des sûtras! Je vais te montrer mon exécrable vie, je vais te révéler mon vrai visage ! ", et sur ces imprécations, il disparaît.

Acte II, scène I. Au palais : cérémonie de mariage de l'empereur Toba et de Taikenmon-in. Au fond de la scène a lieu la dernière danse de bugaku.

La fille adoptive préférée de Shirakawa, Taikenmon-in, dix-huit ans, se marie avec l'empereur Toba, seize ans. Shirakawa, soixante-douzième empereur, avait abdiqué en faveur de son premier fils l'empereur Horikawa pour entrer en religion, mais ce dernier mourut à l'âge de vingt-huit ans et ce fut Toba, premier fils de Horikawa et donc petit-fils de Shirakawa, qui accéda au trône. L'empereur Shirakawa ouvrit ainsi le système de gouvernement par des empereurs retirés ou insei.

Scène de début de banquet et divertissement nocturne. Shirakawa est en compagnie de Fujiwara Yorinaga, frère cadet du premier ministre, qu'il apprécie pour son intelligence et sa perspicacité. Shirakawa lui promet, de façon énigmatique, le poste de kanpaku (grand chancelier) au service de l'empereur qui va naître.

Entrée de Toba et de l'impératrice. On sert du saké parfumé et le banquet s'anime. Chant et danse d'imayō. L'empereur Toba, à qui incombe la responsabilité du service religieux nocturne, quitte le banquet tandis que l'empereur Shirakawa entraîne Taikenmon-in dans ses appartements. 
Acte II, scène II. Devant le jardin qui donne sur la chambre à coucher de Shirakawa.

L'empereur et Taikenmon-in sont dehors, devant la chambre. Ils écoutent les cris des insectes nocturnes et admirent le clair de lune. Ils échangent des poèmes et Shirakawa, prenant la main de la jeune fille, l'entraîne vers l'intérieur de la chambre. Leurs silhouettes enlacées se détachent en ombre chinoise au travers d'un porte-kimono.

\section{Acte II, scène III. Dans le palais de l'empereur Toba.}

Dix ans ont passé. Après la mort de Shirakawa, Toba gouverne en tant que régent secondant l'empereur Sutoku. Cependant, Toba est rongé par la douleur et nourrit une immense rancœur d'avoir été dépossédé du trône impérial par Shirakawa au profit de Sutoku. Or, en l'an cinq de l'ère Hōen (1141), sa deuxième épouse Bifukumon-in lui donne un fils, Konoe Narihito. Aussitôt Toba reporte toute son affection sur ce nouveau petit prince. II renonce alors à protéger Sutoku, qui n'était pas de son sang, et le destitue pour confier le pouvoir impérial à Konoe. Comme celui-ci n’a pas même deux ans, c'est Toba qui assure le gouvernement de l'empire. Cette querelle de succession fut à l'origine des troubles dits de Hōgen.

Dans le Palais. Le petit Konoe Narihito est dans son berceau et deux nourrices lui chantent une berceuse. Elles semblent s'ennuyer et se mettent à jouer à jan-ken-pon (en figurant avec leurs doigts, au hasard, "pierre, feuille, ciseaux »). Entre alors Bifukumon-in. Elle s'approche du berceau de son fils et se met à chanter avec amour "mon petit, sois sans crainte, je te ferai empereur, je te ferai empereur... ». Au centre, l'empereur Toba, absorbé par quelque affaire, paraît préoccupé. En fait, il est poursuivi depuis plusieurs nuits par un horrible cauchemar où des gens du Palais sont emportés par les tourbillons d'un vent violent, qui ressemblent à d'immenses vagues. Il assiste impuissant à leur noyade, car il ne peut attraper que du vide, hélas, seulement du vide. Puis, de grosses taches noires apparaissent dans le ciel, elles grossissent et fondent sur lui. La vision se précise : c'est une troupe de cavaliers armés jusqu'aux dents qui l'attaque. Commence alors un chant traduisant cette sinistre vision maléfique.

L'impératrice parle elle aussi de mauvais présages. Ces temps derniers, elle aperçoit des étoiles filantes : "Ne serait-ce pas quelque effet du gouvernement de Sutoku ? " demande-t-elle, en formant le souhait que Narihito devienne empereur et construise un monde de paix. Mais, par malheur, la 
maladie emportera prématurément le jeune empereur à l'âge de seize ans. Sa mère y verra l'effet d'un sort jeté par Sutoku, ce qui envenimera la guerre entre les deux clans impériaux.

\section{Acte II, scène IV. Dans la salle de réception du même Palais.}

Sutoku, qui vient d'apprendre sa destitution, arrive au Palais en compagnie du ministre de Gauche Yorinaga. Ce dernier presse Toba de lui faire connaître quelle faute leur vaut d'être écartés du pouvoir. Taikenmon-in, première épouse de Toba et mère de Sutoku, défend la légitimité de Sutoku, en alléguant que Narihito est le fils de la deuxième épouse. Toba, qui jusque-là avait gardé le silence, déclare qu'en réalité Sutoku n'est pas son véritable fils mais le fruit de l'union adultérine de Shirakawa et de sa propre femme, Taikenmon-in.

Taikenmon-in s'apprête à protester quand Bifukumon-in arrive avec un pli urgent du directeur des Affaires intérieures dont elle donne lecture. Le moine Shōson aurait avoué avoir jeté un sort maléfique à Narihito sur l'ordre de Yorinaga. À cette nouvelle, Toba accuse Yorinaga de complot et ordonne son bannissement, avec interdiction de sortir de chez lui jusqu'à la décision de la Chancellerie. Tandis que Sutoku, Taikenmon-in et Yorinaga, frappés de stupeur, demeurent sur scène, atterrés à l'idée que la guerre est désormais inéluctable, Toba, Bifukumon-in et le ministre Tadamichi se dirigent rapidement vers le fond et sortent.

\section{Acte II, scène V. Décor de champ de bataille.}

La succession de Konoe-in provoque une rivalité grandissante entre Toba et Sutoku. Ce dernier espérait encore gouverner soit en retrouvant le trône, soit à titre d'empereur retiré si son premier fils Shigehito devenait empereur. Mais Toba préfere laisser l'empire au frère cadet de Sutoku, Masahito, son quatrième fils (plus tard connu sous le nom d'empereur Go-Shirakawa). Ainsi, Sutoku et sa descendance sont-ils définitivement écartés du pouvoir. À la mort de Toba, en 1156 (première année de l'ère Hōgen), va s'ouvrir une guerre de succession opposant Bifukumon-in et l'empereur Go-Shirakawa à Sutoku. Sans attendre, les femmes du clan de l'impératrice passent à l'attaque et incendient le palais de Sutoku, tandis que se déchaîne une guerre fratricide où le sang coule entre parents et enfants, entre aînés et cadets, entre oncles et neveux. La bataille est représentée par un ballet de cavaliers et de soldats qui tirent à l'arc et qui jouent de la lance et du 
sabre. Encerclé, Sutoku doit capituler avant même que les renforts armés n'arrivent de Nara.

\section{Acte II, scène VI. Champ de bataille : défaite de Sutoku.}

Un premier soldat accourt dans le camp de Sutoku pour l'informer de la mort de Yorinaga, puis arrive un second soldat qui lui annonce la destruction par le feu de son Palais. Alors, Sutoku, reconnaissant sa défaite, ordonne aux deux soldats de s'en aller au plus vite et de préserver leur vie pour transmettre à tous ses dernières volontés. Quant à lui, il va se couper les cheveux et se retirer au temple Ninna-ji.

Acte II, scène VII. Jugement de Sutoku et destruction de son clan.

L'impératrice Bifukumon-in réclame un châtiment à la hauteur de la haine qu'elle voue à Sutoku, pour avoir, selon elle, provoqué par des maléfices la mort de son enfant chéri Konoe et de son père l'empereur Toba. Elle obtient que Sutoku soit banni et exilé dans l'île de Naoshima en Sanuki; que Taikenmon-in soit recluse à Sagano; que le premier fils de Sutoku, Shigehito, abandonne le monde, se coupe les cheveux et entre en religion ; que Minamoto no Tameyoshi et tous ses enfants, en bas âge, soient condamnés à la peine de mort; et que tous les enfants de Yorinaga, mort sur le champ de bataille, soient exilés. C'est ainsi que l'on confisqua le patrimoine de centaines et de centaines de personnes et que l'on dispersa leurs familles. Dans ce drame d'une immense ampleur, on vit s'entretuer pères et fils, oncles et neveux, amis et amis...

Acte III, scène I. Devant le mausolée de l'empereur Sutoku, comme au premier acte.

Le fantôme de Sutoku réapparaît à Saigyō : la haine vindicative que lui a inspiré le monde ici-bas était si forte qu'elle a continué même après sa mort, le transformant en une divinité maléfique. Ainsi avoue-t-il : «toutes ces morts cruelles, tous ces désastres, tout cela est de mon fait ".

Le moine Saigyō continue à prier ardemment : «Coupez les liens d'avec le monde des humains, sale et boueux, afin d'accéder au plus vite au paradis de Bouddha". Mais le spectre de Sutoku lui répond: "Toi, tu as choisi la voie de Bouddha, mais moi j'ai choisi la voie inverse, qui m’éloigne de Bouddha, de tout salut et de toute rédemption. Je resterai éternellement 
ici-bas et condamné à revenir sans cesse, je répondrai au mal par le mal, à la haine par la haine et à la force par la force. Tel est le chemin que j'ai choisi ». Après avoir ainsi parlé, Sutoku se transforme en horrible démon.

Acte III, scène II. Épilogue : devant le même mausolée.

Saigyō, qui s'était endormi d'épuisement, le visage tourné contre un rocher, se réveille en s'écriant : "Quel affreux cauchemar! Ah! Puisse son altesse Sutoku être délivrée de la haine qui l'a emprisonnée dans sa vie antérieure, malgré les dix mille cavaliers qui l'entouraient ! Puisse-t-elle cesser d'engendrer malheurs, horreurs, aversions, complots et guerres, sans le moindre instant de repos. Que Bouddha, dans sa grande miséricorde, le conduise vers son paradis..."

Saigyō se recueille et récite le sûtra d'Amida et le chœur se joint à lui.

Le rideau tombe.

\section{Personnages}

SAIGYō (baryton)

Après avoir appartenu à la garde rapprochée de l'Empereur, a quitté sa famille et renoncé à la vie laïque pour devenir bonze, en proie à de nombreux questionnements qui le ramènent au mausolée de Sutoku. Ce fut un religieux et un poète hors du commun.

SuToku Akihito (ténor)

Soixante-quinzième empereur (1119-1164). Il apparaît d'abord sous les traits d'un bûcheron, puis sous ceux du spectre de l'empereur Sutoku. À l'acte II, il revient en tant qu'empereur avant de réapparaittre sous l'aspect du spectre de Sutoku à l'acte III. C'est le personnage principal de ce drame musical.

Devenu empereur à l'âge de cinq ans, il fut destitué à vingt-six ans et l'empereur Toba mit définitivement fin à sa lignée en écartant du trône son fils, Shigehito. Selon la coutume de l'époque, c'était le père qui exerçait le pouvoir à la place de l'empereur enfant. Quand Shirakawa était en religion avec le statut d'Empereur retiré, l'empereur Toba avait gouverné au lieu de Sutoku, tout en sachant pertinemment que celui-ci n'était pas son vrai fils. On aurait pu alors penser que l'empire allait revenir à Sutoku et 
à sa descendance. Mais quand Toba eut un nouveau fils, Narihito, avec sa deuxième épouse Bifukumon-in, qui fut son véritable amour, il écarta autoritairement Sutoku du trône pour y placer Narihito. Puis, à la mort prématurée de ce dernier, Toba accéda au désir de Bifukumon-in et nomma empereur son quatrième fils Masahito, le frère cadet de Sutoku, évinçant ainsi définitivement ce dernier et sa descendance. Le clan de Sutoku se révolta, entraînant la guerre civile de Hōgen, mais subit une dure défaite. Ses fidèles furent décimés, et Sutoku, alors âgé de quarante-cinq ans, fut exilé sur l'île de Naoshima dans la province de Sanuki. Il y mourut dans la solitude, rongé par une haine tenace. Son mausolée se trouve à Shiramine, dans l'île de Shikoku, lieu éponyme de ce drame musical.

ShiraKawa Sadahito (basse baryton)

Soixante-douzième empereur du Japon (1053-1129), il abdiqua en 1086 en faveur de son fils Horikawa et entra en religion. Mais Horikawa mourut à l'âge de vingt-huit ans et ce fut son premier fils, Toba, petit-fils de Shirakawa, qui monta sur le trône à l'âge de quatre ans. Shirakawa, bien que retiré, assura le gouvernement, inaugurant ainsi le système des empereurs retirés ou insei. La fille adoptive de Shirakawa, Taikenmon-in devint la première épouse de Toba. Shirakawa destitua Toba (vingt ans) au profit de Sutoku, car, selon une première tradition que suit ce drame musical, Sutoku était le fils de Shirakawa (qu'il aurait conçu à l'âge de soixante-six ans) et de Taikenmon-in. Une autre tradition rapporte que Sutoku était le fils de Saigyō et de Taikenmon-in.

\section{Yorinaga (ténor)}

Membre de la famille Fujiwara (1120-1156), il fut ministre de Gauche (de l'Est) de l'empereur Sutoku. Son frère aîné Tadamichi était au service de l'empereur Toba. La rivalité successorale entre les deux frères fut une des causes des troubles de Hōgen. Yorinaga, féru de sciences, était d'une intelligence supérieure et d'un grand sérieux, dépassant en tout son frère aîné. Selon le Dit de Högen, Yorinaga fut blessé par une flèche en combattant et mourut quelques jours plus tard. Mais, ici, il trouve une mort dramatique sur le champ de bataille. 
TовA Munehito (haut baryton ou ténor)

Soixante-quatorzième empereur (1103-1156), il accéda au trône à l'âge de quatre ans, à la mort de son père, l'empereur Horikawa. À seize ans, il épousa Taikenmon-in, alors âgée de dix-huit ans. C'est à leur premier fils, Sutoku, dont il n'était pas le vrai père, que revint l'empire. Mais il le destitua pour mettre sur le trône Konoe Narihito, le fils que lui donna sa seconde épouse, Bifukumon-in, qu'il chérit particulièrement. Ce fut une des causes de la guerre civile de Hōgen. Toba était un excellent joueur de flûte de gagaku.

\section{TAIKEnMON-In Shōshi (soprano)}

Fille adoptive de l'empereur Shirakawa (1101-1145), elle devint la première épouse de Toba à l'âge de dix-huit ans. Mère de Sutoku et du futur empereur Go-Shirakawa, elle mourut avant la guerre civile de Hōgen. Mais ici, pour accentuer l'effet dramatique, elle s'oppose à Bifukumon-in, en prenant le parti de Sutoku.

\section{Bifukumon-In Tokushi (soprano)}

Deuxième épouse de Toba, qu'il aima passionnément (1117-1160). De leur union naquit un fils, Konoe Narihito (1139-1155), dont Toba fit, à deux ans, le soixante-seizième empereur en écartant Sutoku. Mais Konoe mourut prématurément à l'âge de seize ans, un an avant son père. Bifukumon-in accusa alors Sutoku d'avoir provoqué la mort de son fils par des maléfices et en conçut une immense haine qui la poussa à écarter Sutoku et sa descendance du pouvoir. À cet effet, elle obtint que Toba, dans ses dernières volontés, nommât comme empereur Masahito, son quatrième fils, au lieu du fils aîné de Sutoku, Shigehito. Ce fut l'étincelle qui alluma, en 1156, les feux de la guerre civile de Hōgen. Toutefois, il serait injuste d'attribuer à Bifukumon-in la principale responsabilité de ces troubles. Aveuglée par un amour maternel passionné, elle ne fit qu'exploiter en faveur de son fils un système de succession impérial arbitraire et l'ambition des grandes familles au pouvoir. Ainsi derrière la brillante culture de la cour de Heian, apparaît la face cachée d'une société où la soif du pouvoir, la haine et la vengeance nourrissent des complots et engendrent des assassinats. 
TADAMICHI (baryton)

Grand Chancelier de Toba, frère aîné de Yorinaga, mais né d'une mère différente, il est en conflit avec son cadet depuis que leur père, Fujiwara no Tadazane, qui préférait Yorinaga, avait octroyé à ce dernier le droit de lignage. Leur rivalité fut aussi une cause des troubles de Hōgen. Quand Yorinaga maria sa fille adoptive Tashi, âgée de huit ans, au petit empereur de neuf ans Konoe, Tadamichi l'imita aussitôt en donnant également sa fille adoptive comme épouse à Konoe. Tadamichi fut un calligraphe renommé.

Go-Shirakawa Masahito (baryton)

Soixante-dix-septième empereur (1127-1192). Dans cette pièce, il n'apparaît que dans la scène du jugement (acte II, scène VII). Monté sur le trône dans un contexte aussi trouble, Go-Shirakawa, en habile homme politique, combattit de sa retraite la classe guerrière émergente.

Le Chambellan impérial de Shirakawa et de Toba (baryton) Joué par le même acteur.

Deux nourrices (alto et soprano) du petit empereur Konoe.

Deux soldats (baryton et ténor) du clan de Sutoku. 


\section{Livret}

\section{Acte I, scène I (prologue)}

Prélude. Le rideau se lève sur le moine Saigyō, il est devant un second rideau intérieur translucide derrière lequel est installé le chœur. Debout devant le mausolée délabré de l'ancien empereur Sutoku, à Shiramine, dans la province de Sanuki sur l'île de Shikoku, Saigyō récite des sûtras tout en marquant la mesure au wood-block. Le rideau translucide donne, même en plein jour, une impression d'obscurité, de brume et de forêt profonde. La voix de Saigyō résonne dans les montagnes. Le moine est au pied du tertre sur lequel est construit le mausolée, devant la clôture en ruine. Descendant de cette éminence, un bûcheron arrive, tout en chantant à l'unisson de la lecture de Saigyō. Le chœur les rejoint pour entonner le sûtra d'Amida. Ce bûcheron n'est autre que la réincarnation de Sutoku.

SAIGYō, récitant le sûtra. - Ainsi, l'ai-je entendu. Un jour le Bouddha se trouvait dans le monastère de la Futée de Jeta, dans les jardins d'Anathapindada au pays de Shravasti, avec 1250 de ses disciples. Tous étaient de grands arhat, dont les noms sont connus de tous. Le vénérable aîné Shariputra et une cohorte de vénérables disciples.

Le Cheur (basse et ténor), reprenant en canon les mêmes paroles. - Ainsi, l'ai-je entendu. Un jour le Bouddha se trouvait dans le monastère de la Futée de Jeta, dans les jardins d'Anathapindada au pays de Shravasti, avec 1250 de ses disciples.

Alto 2. - Écoute, ô toi, vénérable Shariputra. Au Paradis, avec sa septuple enceinte de pierre, son septuple rideau de perles, sa septuple rangée d'arbres, tout est fait des quatre joyaux.

Alto 1. - Dans ce Paradis du Bouddha, on joue toujours de la musique céleste. Le sol y est d'or. Six fois par jour, matin et soir, tombe une pluie de fleurs de mandala.

Soprano 2. - Les lotus bleus émettent des reflets bleus, les jaunes des reflets jaunes, les rouges des reflets rouges et tous exhalent des parfums purs et subtils ; c'est le Paradis d'Amida.

Soprano 1. - Dans ce Paradis se trouvent toujours de nombreuses sortes d'oiseaux rares aux couleurs multiples; six fois par jour, matin et soir, ils entonnent des chants doux et raffinés. 
(L'éclairage varie dans des tons paradisiaques. Les formes aussi varient pour créer des motifs abstraits.)

\section{Acte I, scène II}

Toujours devant le mausolée; le rideau translucide se lève.

LE BûCHeron. - Oh ! Quelle joie que ces prières pour le repos des trépassés offertes sur ce tombeau qu'aucun pèlerin ne visite! Qui es-tu honorable moine à être venu célébrer un office?

SAIGYō. - Je suis un religieux nommé Saigyō, qui parcours tout le pays. Je suis venu cette fois de loin jusqu'à ce mausolée de l'empereur Sutoku, prier pour le repos de son âme, ici à Shiramine dans cette province de Sanuki. Et toi qui apparais ainsi, venu de nulle part, qui donc es-tu?

Le BûCheron. - Je suis un bûcheron qui vis dans les environs, au fond de ces sombres montagnes de Shiramine, dans ces forêts serrées de pins et de chênes où même en plein jour la lumière ne parvient à percer. Oh ! Quelle joie que tu sois venu offrir tes prières dans ce mausolée couvert de végétation où personne ne vient prier. Oh! Oh! Oh! Oh!

SAIGYō. - Bûcheron, tu ne donnes pas l'impression d'être une personne du commun. Aurais-tu peut-être été au service de l'empereur Sutoku ?

Le Bûcheron. - Oui, en effet, et je suis quelque peu au courant de ce qui s'est passé.

SAIgyō. - Alors si c'est le cas, narre-moi en détail l'histoire de Sutoku.

Le BûCHeron. Il descend et se rapproche peu à peu de Saigyō. - Alors, voilà : Sutoku, le soixante-quinzième empereur, est né en l'an deux de l'ère Gen.ei, comme premier fils de l'empereur Toba. Prénommé Akihito, il a grandi en bonne santé, chéri par sa mère Taikenmon-in. Le prince Akihito fut spécialement adoré par son aïeul, l'empereur retiré Shirakawa, qui le fit monter sur le trône à l'âge de cinq ans seulement. Un règne de dix-huit ans, dixhuit ans de paix et de prospérité sur tout l'empire, un règne juste dont tout le peuple se félicitait. Pourtant, ah ! pourtant ! la première année d'Eiji, son père, l'empereur retiré Toba, l'obligea, bien qu'il n'eût commis aucun crime, à quitter le trône pour y mettre son fils le prince Konoe Narihito, âgé alors d'à peine deux ans. Depuis lors, l'empire est sens dessus dessous comme un embrouillamini de fils de lin, la capitale est pleine de guerriers montés, les feux des batailles embrasent la région de Kyōto. Mais ce n'est pas tout! N'étant peut-être pas agréé par le Ciel, le prince Konoe tomba malade peu 
après avoir accédé au trône et mourut malgré son jeune âge. Cependant, sa mère, Bifukumon-in, attribuant cette disparition aux maléfices de Sutoku, en fit part à l'empereur retiré Toba. Ce dernier, probablement aussi parce qu'il était profondément affecté, s'affaiblissait de jour en jour et, malgré les rites et les prières, malgré les traitements et les médicaments, finit par succomber le deuxième jour du septième mois de la première année de Hōgen. Mais Bifukumon-in attribua également cette mort à Sutoku et, le maudissant toujours davantage, invoqua les dernières volontés de Toba pour faire écarter de la succession l'héritier légitime, le prince Shigehito, fils aîné de Sutoku, et mettre sur le trône le quatrième fils de l'empereur retiré Toba, le prince Masahito. Il est clair que cela visait à empêcher que Sutoku ne puisse prendre le titre d'Empereur retiré. Comme le dit le proverbe, ces désordres contre-nature annoncent inévitablement la ruine du pays. Cela n'était certes pas le vœu de Sutoku ; pourtant il a entrepris de se battre uniquement afin d'obéir au Ciel et de répondre aux attentes du peuple, mais hélas, il a été vaincu ; refugié dans le temple de Ninna-ji, il a pris la tonsure et fait preuve d'humilité, mais la haine de Bifukumon-in restait toujours aussi profonde et il a fini par être exilé sur un îlot de la province de Sanuki. SAIGYō. - Ah ! quelle triste histoire que voici ! Que cela tourne ainsi, c'est que l'être humain ne peut échapper aux passions, c'est la loi karmique. Mais alors, comment l'empereur a-t-il vécu dans cet endroit perdu loin de la capitale?

Le Bûcheron. - Il était enfermé sur cet îlot inhabité de Naoshima, au large de Sanuki, sans personne s'affairant à son service. Le bruit des insectes qui se lamentent dans les haies, le cri des pluviers qui s'agitent sur le promontoire alourdissaient ses tourments. Empli de nostalgie, il ne cessait de penser à la vie animée de Kyōto. Alors pour exaucer son vœu de rentrer à la capitale, il fit couler du sang de son doigt et, pendant trois ans, copia les cinq sûtras du Grand Véhicule qu'il envoya à la cour de l'impératrice avec un poème :

\footnotetext{
Pluvier des plages, pluvier des plages, tes traces se transmettent à la capitale.

Ohoo! Ohoo!

Mais ici à Matsuyama, mon corps ne peut que gémir.

Ohoo! Ohoo!
} 
Cependant, l'empereur et tout le gynécée virent en ces sûtras un maléfice et en firent renvoyer les copies. Sutoku en fut plein de ressentiment : «Que c'est mortifiant! Si même ces sûtras sont rejetés, alors à quoi bon survivre davantage? " Sur ce, il ne coiffa plus sa chevelure, ne coupa plus ses ongles, et se transforma de son vivant en un démon! Ah, quelle chose pitoyable! Et ce n'est pas tout, car Sutoku se coupa le bout de la langue et, avec le sang qui en coulait, inscrivit sur sa copie du Grand Véhicule un serment exprimant sa volonté : «Ahaa! Ahaa! Oui, voici ma volonté, voici mon désir : devenir un grand démon sur cette terre et faire de ce monde le champ de sanglantes batailles! » Puis il précipita le document au fond de la mer et, après avoir passé neuf ans de souffrances, finit par disparaître. Probablement à cause de la profondeur de ses pensées, les volutes de fumée de la crémation s'étirèrent en direction de la capitale. Ah, quelle chose effroyable!

SAIGYō. - Quel destin cruel à entendre ! Mais comment se fait-il que tu connaisses avec tant de précision le sort de Sutoku?

Le BûCheron. - Pourquoi devrais-je encore te le cacher ? Je suis l'esprit de Sutoku, le soixante-quinzième empereur. Je me suis manifesté devant toi pour te remercier de tes prières et, maintenant, je vais te montrer ma vraie forme.

\section{Acte I, scène III}

Même décor devant le mausolée; la musique se fait plus forte. Entre-temps, le bûcheron se transforme en Sutoku sous son effroyable forme démoniaque, à la longue chevelure désordonnée et aux ongles démesurés.

Sutoku. - Ainsi donc, Saigyō, tu es venu de la lointaine capitale dans l'intention de prier pour le repos de mon âme, quelle bonté extrême ! Mais pourtant, vouloir faire de moi un bouddha par les mérites de la récitation des sûtras, n'est-ce pas une entreprise insensée?

SAigyō. - En effet, mais comment avez-vous pu vous perdre ainsi ? Renoncez donc à cet attachement à un monde complètement souillé et accédez au stade apaisé de l'Éveil !

Sutoku. - Tu ne peux pas le savoir, mais ce que je t'ai raconté tout à l'heure n'est que la surface des choses. Saigyō, regarde bien l'état véritable de ma vie, regarde la profondeur de mon karma. Regarde, Saigyō ! Regarde ! (L'écho retransmis par haut-parleurs se diffuse dans la salle.) 


\section{Acte II, scène I}

La cour impériale, la scène devient plus lumineuse, plus vaste. Cérémonie d'entrée à la cour de Taikenmon-in (18 ans), future épouse de l'empereur Toba (16 ans). Au fond de la scène, quatre danseurs jouent le morceau de bugaku "Seigaiha" qui clôt la cérémonie. Pendant ce temps, venant du centre, des courtisans entrent en scène. Sur le devant de la scène, des dames de cour (chour de femmes) sont en train de parler.

Le Chenur de femmes. - Félicitations pour ce jour de fête! Que vienne un règne plein de sagesse, un règne de mille automnes, un règne de dix mille ans! Le Paradis est déjà ici, sur cette terre! Le Paradis est déjà ici, sur cette terre!

SHIRAKaWA, empereur retiré entré en religion. - Alors Yorinaga, que pensezvous de cette cérémonie?

Yorinaga. - C'est le fondement d'une lignée impériale de dix mille ans ; le départ, je pense, d'un règne encore plus glorieux que celui des anciennes ères d'Engi et de Tenryaku.

ShirakaWA. - Voilà qui est bien parlé. Alors, je vous confère le titre de Grand Chancelier.

YorINAGA. — Quoi ? Chancelier! (parlantrapidement)Vous voulez dire celui de l'empereur Toba? Je suis bien trop jeune, je ne peux même pas y penser. ShirakaWa. - Non, il ne s'agit pas du chancelier de l'empereur Toba, mais de celui du prince, son fils.

Yorinaga. - Quels étranges propos! Vous dites des choses incompréhensibles puisque l'empereur Toba n'a pas encore d'enfant !

ShirakaWa. - Bien sûr, vous ne pouvez pas le savoir. Mais moi, je peux de mes yeux lire le futur dans la paume de ma main.

Le Chambellan. - Je vous annonce que leurs Majestés impériales sont arrivées.

Shirakawa. - Qu'on les fasse entrer.

Le Cheur, mixte, divisé en six parties. - L'empereur Toba, petit-fils légitime de l'empereur retiré ! Sa femme, la princesse, fille adoptive de l'empereur retiré qui l'a élevée avec amour. Qu'ils coulent de longs jours en parfaite harmonie!

(Toba, Taikenmon-in entrent solennellement sur la scène en tenue de mariage, avec leur suite.) 
Това. - La cérémonie s'est parfaitement bien déroulée. Tout cela uniquement grâce à Votre bienveillance.

ShirakaWA. - Félicitations! Je vous souhaite de rester toujours unis. Et vous, Madame, comment vous sentez-vous?

(On fait circuler des coupes de saké de cérémonie.)

TAIKENMON-IN. - Je suis très honorée d'être née sous Votre glorieux règne. ShirakaWa. - Certes, mais il ne faut pas oublier, ne serait-ce qu'un instant, la protection du Bouddha. Pour ce banquet de célébrations, divertissons-nous un peu en chantant des poèmes imayō. Que l'empereur Toba prenne sa flûte et que Taikenmon-in et Yorinaga se joignent à nous, moi aussi je vais chanter.

(On joue alors un imayō sur la mélodie de "Etenraku »)

À l'aube du nouveau printemps,

Regardant les montagnes qui nous entourent,

Fleurs de cerisiers épanouies !

Pas un pic qui ne soit plongé dans un nuage blanc!

(Tous dansent et chantent en improvisant des gestes, chacun de son côté.) Chambellan. - Il est déjà minuit passé, il est temps que Sa Majesté regagne la Cour.

ShirAKaWA. - Mais nous ne sommes encore qu'au milieu de cette nuit de fête!

ТовА. - Oui, c'est vraiment dommage, mais il me reste des rites nocturnes à accomplir, aussi je vous demande l'autorisation de me retirer.

ShIrAKaWA. - Il ne faut pas négliger ces obligations du soir, je suis heureux de voir que vous y veillez avec une grande attention. Alors, retournez sans crainte à la Cour.

(Toba et Taikenmon-in se lèvent et s'apprêtent à partir.)

Un instant! Un instant! Toba a des rites sacrés à accomplir, mais vous, Madame, n'en avez pas, aussi pouvez-vous rester encore un moment à converser avec moi. Les célébrations de cette nuit sont loin d'être achevées ; alors, qu'en pensez-vous Madame?

TaIKenMon-IN. - Vos paroles m'honorent, mais ce soir, c'est la cérémonie de mon entrée à la Cour, je vous prie donc de m'excuser.

Това. - Comme c'est la proposition de mon auguste grand-père, il vous faut l'accepter. Restez donc un moment en sa compagnie. 
(Toba regagne le palais, tous quittent la scène. La scène tourne et laisse apparaître une pièce qui donne sur un jardin intérieur.)

\section{Acte II, scène II}

Donnant sur un jardin intérieur, la chambre à coucher de l'empereur retiré. Shirakawa et Taikenmon-in écoutent les cris des insectes. Musique calme.

ShirakaWA. - Les nuages cachent la lune.

Taikenmon-In. - Les lucioles voltigent et se croisent, c'est bientôt l'automne. L'étoile du matin pâlit, la nuit se fait plus longue.

(Shirakawa prend la main de Taikenmon-in, ils entrent dans la pièce. Le couple enlacé se profile en ombre chinoise sur le paravent porte-kimono de la chambre. La musique se fait plus forte. Le couple se laisse tomber sur la couche. La musique se calme, indiquant le passage du temps.)

(Un panneau apparaît : "10 ans plus tard, l'empereur retiré Shirakawa décède à l'âge de 76 ans ».)

\section{Acte II, scène III}

Le palais de l'empereur retiré Toba.

Le Cheur, six parties. - L'empereur Toba a accédé au trône à l'âge de cinq ans. Son règne a duré seize ans, un règne épargné par les malheurs et conforme au déroulement correct des quatre saisons. Depuis la disparition de Shirakawa, l'empereur retiré Toba gouverne le monde, l'empire est prospère et partout règne la grande charité du Bouddha. Cependant, la cinquième année de l'ère Hōen, Bifukumon-in a donné naissance au prince Narihito. Désormais Toba, qui le chérit par-dessus tout, s'emploie à chasser Sutoku du trône pour y mettre ce fils, âgé d'à peine deux ans, et les relations entre les deux hommes vont se dégrader.

(L'intérieur du Palais; autour du berceau du prince Naribito, ses deux nourrices chantent une berceuse.)

Les nOURRICES. - Do, do, dormez ! Dormez petit prince, dormez en paix. Ouverte, votre main est une feuille d'érable rougie; fermée, une petite pierre. Do, do, dormez! Dormez petit prince, dormez en paix. Grandissez sain et vigoureux. Votre oreille est comme une feuille de ginkgo, votre nez, comme une cerise. 
(Les nourrices jouent à jan-ken-pon, "pierre, feuille, ciseau ») Les ciseaux coupent. Le caillou frappe. À toi de jouer ! Hoï ! Hoï ! Hoï ! Hoï ! Hoï ! Hoï ! Hoï ! Hoï ! J’ai gagné ! Hoï ! Match nul ! Hoï ! J’ai gagné ! (La perdante se fait tirer l'oreille) Ouille, ça fait mal ! Le caillou frappe. À toi de jouer ! Hoï ! Hoï ! Hoï ! Hoï ! Hoï ! Hoï ! Hoï ! Hoï ! J’ai gagné ! Hoï ! Match nul ! Hoï ! J’ai gagné !

Bifukumon-IN. — Quel bruit vous faites! Vous allez réveiller mon fils! Toi mon seigneur, toi que j'ai mis au monde, si je ne parviens pas à te faire monter sur le trône, alors ton destin sera de passer ta vie dans l'obscurité, sans voir la lumière. (Elle se penche sur le berceau, et tout en le berçant, chante) Dors, dors, dors, dors mon petit prince! Dors en paix. Moi, je te ferai absolument accéder au trône. (Elle regarde la lumière au fond de la pièce) Mon seigneur semble être encore en train de s'occuper des affaires de l'État. (La scène tourne: apparaît Toba, au centre, en train de lire; il semble fatigué et préoccupé par quelque chose.) Je me demande s'il n'y a pas quelque chose qui le contrarie.

Това. - Cela fait dix ans que je m’occupe des affaires de l'État. Pendant tout ce temps, obéissant à la volonté du Ciel, j'ai récompensé les justes et apaisé les mauvais. Mais je n'arrive pas à me débarrasser d'un rêve de mauvais augure.

Bifukumon-In. - On dit que les cauchemars viennent de la fatigue des cinq viscères. Reposez-vous un moment, afin d'apaiser votre cœur. Cependant, quel est donc ce cauchemar? Ne voulez-vous pas m'en faire part ?

Това. - C'est un rêve qui revient sans cesse : comme noyés par les vagues, les courtisans du Palais sont emportés par le vent, mais j'ai beau tendre les mains pour les secourir, je ne saisis que le vide, rien que le vide! Et en plus de cela, il y a des taches noires au centre du tourbillon, des taches qui s'approchent menaçantes, comme des flèches. À regarder attentivement, je comprends que ce sont des cavaliers en grand nombre, les armes à la main, qui se précipitent sur moi comme des guerriers sortis des enfers. Oh! Oh! Oh!

Bifukumon-IN. - Ah ! Mon seigneur bien aimé, quel épouvantable cauchemar! On dit aussi que ces étoiles filantes qui apparaissent souvent depuis quelques temps sont de mauvais présages. Ne serait-ce pas dû au gouvernement de Sutoku ? Si mon fils n'accède pas au trône, alors il sera contraint à passer toute sa vie dans l'obscurité, sans parvenir à la lumière. 
Това. - Oui, en effet. Moi aussi, j’ai vu mon trône usurpé par Sutoku et ne connais depuis que douleurs et humiliations. Je ne peux oublier mes souffrances, pris en étau entre Shirakawa et Sutoku. De plus, ce dernier n'est mon fils que de nom. Il est le fruit des amours adultérins de l'impératrice et de mon grand-père ; aussi maintenant que celui-ci est mort, pourquoi donc devrais-je encore le protéger?

Bifukumon-IN. - Mon seigneur bien aimé, je vous en prie, mettez mon fils sur le trône et faites régner la paix.

Това. - Oui, voilà, c'est cela, je vais faire déposer Sutoku et mettre Narihito sur le trône. Faisons appeler le Grand Chancelier Tadamichi. (Tadamichi arrive précipitamment côté jardin.)

TaDAmichi. - Sire, me voici ! Songez-vous à promulguer un décret? Quelles sont vos instructions?

Това. - Narihito a maintenant deux ans ; pour célébrer cet anniversaire, je veux le faire accéder au trône. Qu'en pensez-vous?

TADAmichi. - Certes, mais qu'avez-vous l'intention de faire de l'empereur Sutoku?

Това. - Donnons-lui un titre d'Empereur retiré !

Tadamichi. - Dans ce cas, nous l'appellerons le « Nouvel Empereur retiré » et vous serez le "Premier Empereur retiré ».

Това. - Ce que je vais maintenant vous dire n'entre pas dans le décret, aussi n'est-ce pas la peine de le mettre par écrit. Mais écoutez mes instructions secrètes : après ma mort, il faut faire monter sur le trône Masahito, le quatrième prince.

Tadamichi. - Bien, bien. J'ai compris votre volonté d'écarter la lignée de Sutoku de la liste de succession. Sur ce point, il faut voir ce que va faire mon frère cadet, Yorinaga.

Le Cheur. - Ah, que les passions humaines sont pitoyables! Ah ! Que la loi karmique est dure! Soif de pouvoir, soif des richesses, concupiscence, fourberie, orgueil, l'homme est captif de toutes ses passions, et se livre sans cesse à la rancœur, aux intrigues et aux massacres. Oui vraiment, quelle tristesse que ce karma humain! Il est clair que c'est là la source des malheurs de ce monde-ci, la source des malheurs du monde à venir. Cette loi karmique des rétributions est la règle immuable de notre monde.

(La musique se fait plus forte.) 


\section{Acte II, scène IV}

Même palais - confrontation entre Toba et Sutoku.

Le Chambellan. - Je vous annonce la venue de Sa Majesté Sutoku et du ministre de Gauche Yorinaga.

TADAMICHI acquiesce d'un signe de la tête après avoir regardé Toba. - Très bien, fais-les entrer. (Sutoku et Yorinaga, l'air excité, entrent d'un pas précipité.) Sutoku. - Père ! Que se passe-t-il ? Je ne comprends pas vos ordres. Cela fait dix-huit ans que je gouverne et pendant tout ce temps-là j'ai agi selon votre volonté et celle de mon aïeul. Et vous aussi devez savoir que j'ai œuvré à la prospérité du pays et fait régner l'ordre et la paix.

Yorinaga, qui essaie de calmer Sutoku d'un geste de la main. - Nous ne pouvons pas accepter ainsi ce décret de destitution, même s'il sort de votre bouche. Faites-nous donc savoir les fautes qui nous sont reprochées. Je suis responsable des erreurs de gouvernement de l'empereur, aussi montreznous où nous avons commis des erreurs et j'accepterai alors le châtiment, quel qu'il soit.

TAIKenMON-In. Elle entre précipitamment, l'air très excité. - Quoi mon seigneur? Je dois m'élever contre cet ordre dépourvu de raison! Vous devez savoir que l'empereur Sutoku, que j'ai élevé, est l'héritier légitime du trône impérial. D’ailleurs, le prince Narihito est né de l'épouse en second.

Yorinaga, qui essaie de calmer Taikenmon-in d'un geste de la main. - II me semble que le prince Narihito n'est pas en ligne de compte pour l'accession au trône. Cependant, si vous tenez vraiment à un changement d'empereur, alors mettez sur le trône le premier fils de Sutoku, le prince Shigehito, car j'estime qu'il serait correct de préserver ainsi la lignée impériale légitime. Néanmoins, il est évident que cette mesure est une intrigue pour empêcher Sutoku de prendre le titre d'Empereur retiré. Alors, dans ce cas, je protégerai Sutoku, même s'il me faut recourir à la force.

Sutoku. - Père, je ne comprends pas votre silence. Dites ce que vous avez au fond du cœur.

Това. - Il s'agit de choses qui se sont passées avant votre naissance, et comme cela ne vous concernait pas, je ne vous en ai pas parlé, mais si vous y tenez, je vais le faire. Écoutez donc calmement. Vous n'êtes mon fils que de nom. En réalité, vous êtes le fils de mon grand-père, l'ancien empereur Shirakawa et de ma première épouse l'impératrice Taikenmon-in ; vous êtes donc le frère cadet de mon père, et par conséquent, mon oncle. 
TaIkenmon-In. - Quoi! Que racontez-vous donc! Sutoku est le fils que j'ai eu de vous!

BifuKumon-IN. Elle arrive précipitamment côté jardin, une lettre à la main. - Mon seigneur bien aimé, regardez donc ce message de Masayori, le directeur des Affaires intérieures, qu'un coursier rapide vient d'apporter. Un maître en exorcisme nommé Shōson a avoué avoir jeté un sort sur le prince Narihito à la demande du ministre de Gauche, son excellence Yorinaga ici présent.

Tова. - Alors Yorinaga ? Avec cela, ta trahison est clairement prouvée ! Tu seras condamné à l'exil, mais en attendant, tu es assigné à résidence jusqu'au résultat de l'enquête de la Chancellerie.

(Toba, Bifukumon-in et Tadamichi sortent précipitamment.)

Yorinaga. - Quel malheur! Je suis pris au piège, comme un lièvre! (Sutoku, Taikenmon-in et Yorinaga restent au milieu de la scène, l'air désespéré.) Désormais, la guerre est inévitable! Oh! Oh!Oh!

\section{Acte II, scène V}

Scène de combats. La musique se fait plus forte, la scène sélargit pour devenir un champ de bataille.

Le Cheur. - Ah ! Quelle chose pitoyable que le destin des hommes! Après la mort de Toba, les luttes de succession aboutissent en l'an un de Hōgen à des combats. Séparés en deux camps, parents et enfants, frères aînés et cadets, oncles et neveux, tous s'entretuent. Quelle situation lamentable! (Scène de combat par le corps de ballet; les poses d'affrontement singulier des cavaliers, maniant l'arc, la lance et le sabre, sont stylisées, évitant tout réalisme. La musique se fait plus forte. Les cavaliers au combat traversent la scène, d'abord de droite à gauche puis de gauche à droite. Avant d'entrer en scène, ils poussent des cris de guerre et on entend le bruit des fers qui se croisent.)

\section{Acte II, scène VI}

Défaite des partisans de Sutoku. Côté jardin, le camp de Sutoku; du centredroite, arrive un guerrier.

GUERrIER 1. - Sire, je viens vous annoncer que son Excellence le ministre de Gauche Yorinaga a été tué au combat par une flèche perdue.

Sutoku. - Quoi ? Yorinaga! 
Guerrier 2, fait son entrée côté cour. - Les flammes gagnent le Palais, il vous faut rapidement quitter ce lieu. Le seigneur Tametomo assure la défense de la Porte occidentale alors que nous protégeons cette partie. Dépêchez-vous de vous éloigner.

Sutoku. - Je suis touché de votre attention, mais puisque j’ai perdu ce combat, il n'y a plus personne susceptible de prolonger ma lignée. Alors, à quoi bon m'enfuir! D'ailleurs, si vous restez tous ici à combattre pour assurer ma fuite, il est clair que beaucoup de sang sera versé inutilement. Du moment qu'on en est là, je ne veux pas entraîner dans le combat les femmes et les enfants, aussi je vais rester ici et attendre le jugement des hauts dignitaires. Alors vous autres, éloignez-vous donc de ces lieux.

Guerriers 1 ET 2. - Notre vie vous est consacrée, pourquoi revenir sur ce point?

Sutoku. - Vos intentions sont louables mais je ne veux pas de massacres inutiles, alors quittez vite ces lieux. Suivez mes instructions en les considérant comme un ordre de l'empereur. Faites savoir partout qu'il faut les considérer comme mon dernier décret impérial.

(Se retournant, les deux guerriers quittent la scène en courant.)

\section{Acte II, scène VII}

Le jugement. Bifukumon-in, Tadamichi, l'empereur Go-Shirakawa et de nombreux autres nobles sont installés sur la partie haute; Sutoku, Taikenmon-in, Shigehito, Minamoto no Tameyoshi et Tametomo ainsi que d'autres guerriers sont dans la partie inférieure.

Le Cheur. - Ah ! Quelle chose pitoyable que le destin des hommes! Quelle chose tragique que le cœur des hommes! La rancœur des femmes est profonde, les châtiments sont lourds!

Bifukumon-In. - Mon seigneur bien aimé, mon époux et mon enfant m’ont déjà quittée. Tout ce qui me reste n'est que haine et rancœur profondes.

Le Chambellan. - Alors, nous allons procéder à la lecture des peines. (Bifukumon-in commence la première la lecture des sentences, suivie d'abord par Tadamichi, puis par Go-Shirakawa, dont les lectures se superposent en canon.)

Bifukumon-in. - Sutoku-in Akihito, pour crime de rébellion, est exilé à l'île de Naoshima en Sanuki et est interdit de retour à la capitale. 
Taikenmon-in Shōshi, pour crime de complicité avec la rébellion, est interdite de séjour à la capitale et recluse à Sagano. Le fils aîné de Sutoku-in, Shigehito, doit prendre la tonsure auprès du révérend Kangyō au sanctuaire de Kazōin, sans retour possible à la vie civile. Minamoto no Tameyoshi est condamné à mort pour avoir participé à la rébellion. Son fils, Tametomo, parce qu'il a fait preuve de grande bravoure au combat, échappe à la peine de mort et est condamné à l'exil sur l'île d'Ōshima en Izu. L'officier de la Porte de droite, Taira no Tokihiro, est condamné à mort pour avoir participé à la rébellion. Exil, peine capitale...

TADAMICHI. — Le ministre de Gauche Yorinaga, pour le crime de rébellion et pour celui d'avoir fait jeter un maléfice au prince Narihito, est condamné à mort. Son fils Kanenaga est exilé pour participation à la rébellion. De même Fujiwara no Moronaga est exilé. Idem pour son fils Fujiwara no Takanaga. L'adjoint à l'Office des chevaux de Gauche, Taira no Tadamasa, condamné à mort pour avoir participé à la rébellion, ainsi que son fils aîné Taira no Nagamori. De même que Taira no Tadatsuna, Taira no Masatsuna, Taira no Michimasa, tous condamnés à mort pour avoir participé à la rébellion. Exil, peine capitale...

Go-Shirakawa. - Minamoto no Kamewaka, lié à Tameyoshi, est, malgré son jeune âge, condamné à mort. De même pour Minamoto no Tsuruwaka, lui aussi impliqué, et pour Minamoto no Ten.ō. Exil, peine capitale... Cheur. - Ah ! Quelle chose pitoyable que les actes des hommes! Quelle chose tragique que la haine des hommes! Ainsi les enfants en pleurs exécutent leurs parents, les cadets sont tués par leurs frères aînés, les amis par leurs amis ; parents et enfants, aînés et cadets, femmes et enfants, tous sont dispersés aux quatre vents! Que tout cela est lamentable!

(La musique se fait plus forte; pendant ce temps, les guerriers vaincus sont emmenés à gauche et à droite, parents et enfants sont séparés.)

\section{Acte III, scène I}

Le mausolée de Sutoku, même décor que l'acte I; après un bref prélude, l'esprit de Sutoku apparaît, sa voix comme dans un écho.

Sutoku. - Saigyō, tu vois donc le karma qui m’a réduit à cette funeste condition. Je suis le fruit de relations coupables, j'ai grandi dans la vanité, la débauche et les intrigues; je suis mort dans la rancœur, dans la haine et la solitude. À cause de la profondeur de mes ressentiments, je n'arrive pas 
à me défaire de cette haine que j'ai envers ce monde et, même après ma mort, je continue à le maudire. Les calamités et les massacres répétés de ces derniers temps, tout cela est mon œuvre.

SAIGYō. - Est-ce que ce sont là des accomplissements dignes d'un sage empereur choisi par le Ciel ? Si un souverain qui doit régner sur le pays dévie de la voie juste des hommes, n'entraîne-t-il pas inévitablement les souffrances du peuple? Je prie pour que vous renonciez à cet attachement à un monde souillé et que vous parveniez à l'Éveil.

Sutoku. - As-tu donc oublié ! Respectant les dieux et les bouddhas, j'ai gouverné selon les intentions du Ciel pendant dix-huit ans; pendant ce temps-là la paix régnait, les récoltes étaient abondantes et le peuple se réjouissait. Mais l'ordre naturel des choses a basculé et, malgré mon innocence, Bifukumon-in m'a fait chasser du trône pour y faire accéder son fils bien aimé Narihito, qui n'avait alors qu'à peine deux ans. Et ce n'est pas tout : mettant à profit la disparition de l'empereur Toba, cette femme a empêché que mon fils Shigehito succède au trône, pour y faire monter mon frère cadet Masahito. Tout cela uniquement pour que je ne devienne pas empereur retiré. En outre, elle a attribué la mort prématurée du prince Narihito à nos maléfices et est allée jusqu'à faire condamner à l'exil un Yorinaga pourtant innocent. Aussi n'y avait-il rien d'autre à faire que d'utiliser la force contre le mal. Et c'est ainsi que les combats ont éclaté. Tameyoshi et son fils ont vaillamment combattu mais la partie était trop inégale ; alors qu'on attendait les renforts envoyés de Nara, l'ennemi a mis feu au Palais; c'est ainsi que, entourés par les flammes et encerclés par l'ennemi, nous avons perdu cette bataille.

SAIGYō. - Quelle pitié m'inspirent vos propos! Mais maintenant que vous n'êtes plus de ce bas monde, pourquoi donc y restez-vous si attaché ?

Sutoku. - Ce doit être à cause de ma haine et de ma rancune !

SAIGYō. - Que dites-vous là ! Haine et rancune sont des choses de ce bas monde ! Dépêchez-vous de renoncer à vos obsessions et d'accéder au monde de l'Éveil!

Sutoku. - Tu ne sais pas tout ! Après ma défaite, j'ai coupé mes cheveux et suis entré en religion ; je ne tenais pas à prolonger ma propre existence, mais je voulais prier pour le repos de ceux qui avaient donné leur vie pour moi. Me sacrifiant, j'ai plusieurs fois supplié que l'on épargne ceux qui s'étaient alliés à moi, mais la haine de cette femme était profonde, et elle a fait exécuter des centaines de personnes, y compris des enfants innocents. 
Et il faut ajouter les centaines de personnes condamnées à l'exil. Et encore les centaines de familles qui ont perdu leur domaine et ont été dispersées. Tout cela ne va-t-il pas contre le Ciel et l'humanité ?

SAIGYō. - Oui, c'est immensément cruel et immoral ! Mais le mal ne peut que conduire au mal, la rancune à la rancune, alors donc renoncez à vos basses passions et remettez-vous-en à la compassion du Bouddha!

SuTOKU. - Quels propos grotesques tiens-tu là! Toi, tu as opté pour la voie du Bouddha, mais moi j'ai choisi ce bas monde des passions! Je demeure sur cette terre, à tourner sans cesse, en dressant le mal contre le mal, la force contre la force, voilà la voie que j'ai choisie! Oho! Oho!

(La musique augmente. Pendant ce temps, l'éclairage clignote violemment, représentant le pouvoir du roi des démons. Puis la musique se calme, la lumière séclaircit peu à peu. Brume matinale, avec des chants d'oiseaux. Saigyō, qui dormait couché sur un rocher, se réveille.)

\section{Acte III, scène II (épilogue)}

Le chour est installé derrière le rideau intermédiaire translucide; Saigyō est devant le rideau.

SAIgYō. Il se lève. - Quel horrible cauchemar ! Ainsi même un souverain, jadis entouré de centaines de serviteurs, reste prisonnier de ses haines, pris dans le monde d'aujourd'hui à errer sans trêve, à courir dans tous les sens comme un guerrier des enfers, à accumuler le mal, la haine, les complots et les conflits sans connaître un instant de repos. Je prie, oui je prie pour que, grâce à la miséricorde du Bouddha, il rompe son attachement aux choses d'ici-bas et qu'il soit conduit dans le monde de l'Éveil.

Le Cheeur, Basse 1. - Ainsi, l'ai-je entendu. Un jour le Bouddha se trouvait dans le monastère de la Futée de Jeta, dans les jardins d'Anathapindada au pays de Shravasti.

BASSE 2. - Les vénérables disciples et d'innombrables saints et divinités se trouvaient dans la foule.

TÉnor 2. - Appelé Amida, il faisait une prédication : ô toi, Shariputra, pourquoi appelle-t-on cette terre le Paradis d'Amida ? C'est que tous les êtres s'y trouvant...

TÉNOR 1. - De là sortent des sons merveilleux et subtils, comme si des milliers d'instruments de musique jouaient ensemble. Tous ceux qui entendent cela, tout naturellement, pensent au Bouddha. 
Atro 1. - Le bleu émet des reflets jaunes, le rouge des reflets blancs. Des parfums purs et subtils. Ô toi Shariputra, ici dans ce Paradis, tout représente l'accomplissement des vertus.

Alto 2. - Tous ceux qui entendent cette prédication veulent renaître sur cette terre, car ainsi ils pourront rencontrer...

Soprano 1. - Vous, tous les êtres sensibles, vous devez croire en ces vertus mystérieuses, les louer et vous confier à ce sûtra vénéré par tous les bouddhas.

Soprano 2. - O Ô vénérable Shariputra, qu'en penses-tu ? Pourquoi est-ce que je dis que c'est le sûtra vénéré par tous les bouddhas?

SAIgYō. - À ce moment-là, le Bouddha dit au vénérable Shariputra: d'ici en direction de l'ouest, après avoir traversé les innombrables terres des bouddhas, se trouve une terre appelée le Paradis de l'Extrême Joie, où réside l'Éveillé. Le bouddha Shakamuni a su accomplir des choses rares et difficiles dans ce bas monde, dans ce monde mauvais des cinq souillures, celles du désir, de la société, de l'opinion, de la brièveté de l'existence et des relations entre les créatures.

(Le chour entonne le sûtra, Saigyō chante avec le chour, et le rideau descend alors que l'orchestre joue plus fort. Est-ce que l'âme de Sutoku a été sauvée par le bouddhisme, ou est-ce qu'il continue à tourner comme un damné exclu de tout salut? C'est à chacun d'en juger.) 